\title{
Decay of mRNAs targeted by RISC requires XRN1, the Ski complex, and the exosome
}

\author{
TAMAS I. ORBAN and ELISA IZAURRALDE \\ EMBL, D-69117 Heidelberg, Germany
}

\begin{abstract}
RNA interference (RNAi) is a conserved RNA silencing pathway that leads to sequence-specific mRNA decay in response to the presence of double-stranded RNA (dsRNA). Long dsRNA molecules are first processed by Dicer into 21-22-nucleotide small interfering RNAs (siRNAs). The siRNAs are incorporated into a multimeric RNA-induced silencing complex (RISC) that cleaves mRNAs at a site determined by complementarity with the siRNAs. Following this initial endonucleolytic cleavage, the mRNA is degraded by a mechanism that is not completely understood. We investigated the decay pathway of mRNAs targeted by RISC in Drosophila cells. We show that 5' mRNA fragments generated by RISC cleavage are rapidly degraded from their $3^{\prime}$ ends by the exosome, whereas the $3^{\prime}$ fragments are degraded from their $5^{\prime}$ ends by XRN1. Exosome-mediated decay of the $5^{\prime}$ fragments requires the Drosophila homologs of yeast Ski2p, Ski3p, and Ski8p, suggesting that their role as regulators of exosome activity is conserved. Our findings indicate that mRNAs targeted by siRNAs are degraded from the ends generated by RISC cleavage, without undergoing decapping or deadenylation.
\end{abstract}

Keywords: argonaute proteins; exosome; mRNA decay; RNA interference; RISC; Ski complex; XRN1

\section{INTRODUCTION}

RNA interference (RNAi) belongs to a family of related processes that lead to the silencing of gene expression by double-stranded RNA (dsRNA) molecules. These pathways act not only at the post-transcriptional level by eliciting mRNA decay or repressing translation, but also at the transcriptional level by inducing heterochromatin formation (for review, see Ambros 2004; Lippman and Martienssen 2004; Meister and Tuschl 2004).

The process of RNAi is triggered by the presence of long dsRNA molecules in the cell. During the initiation step of RNAi, these dsRNA molecules are cleaved into small interfering RNA duplexes (siRNAs) by the RNase III-like enzyme Dicer (Bernstein et al. 2001; Elbashir et al. 2001; Grishok et al. 2001; Hutvágner et al. 2001). The siRNAs are characterized by a 19-20-base-pair (bp) duplex region and a 2-nucleotide (nt) 3' overhang on each strand. During the effector step of RNAi, the siRNAs are incorporated into a multimeric protein complex called RNA-induced silencing complex (RISC), where they serve as guides to select

Reprint requests to: Elisa Izaurralde, EMBL, Meyerhofstrasse 1, 69117 Heidelberg, Germany; e-mail: izaurralde@embl-heidelberg.de; fax: +49 6221387306.

Article published online ahead of print. Article and publication date are at http://www.rnajournal.org/cgi/doi/10.1261/rna.7231505. complementary mRNA substrates for degradation (Hammond et al. 2001; Schwarz et al. 2002).

dsRNA molecules may originate from viral replication, transcription of pseudogenes and repetitive sequence elements, or during transposition of mobile genetic elements. Consequently, gene silencing pathways are thought to contribute to the stability of the genome by silencing the expression of viruses, transposons, and repetitive sequences (for review, see Plasterk 2002; Ambros 2004; Meister and Tuschl 2004). Another endogenous source of dsRNAs results from the transcription of microRNA (miRNA) genes. miRNAs are 19-25-nt-long RNAs produced by cleavage of dsRNA hairpins encoded in the genome (for review, see Ambros 2004). Plant miRNAs are often fully complementary to their targets and elicit mRNA decay (for review, see Baulcombe 2004). In contrast, animal miRNAs are only partially complementary to their targets and do not generally elicit decay but repress translation (for review, see Ambros 2004).

Additionally, long dsRNA molecules can be introduced exogenously. In Drosophila and Caenorhabditis elegans, exogenous dsRNAs are processed by Dicer and enter the RNAi pathway (for review, see Meister and Tuschl 2004). In mammalian cells, exogenous dsRNA molecules elicit an interferon response and lead to a nonspecific degradation of cellular mRNAs and a general inhibition of translation (for 
review, see Williams 1999). However, chemically synthesized siRNAs artificially introduced in the cell enter the RNAi pathway and specifically target complementary mRNAs for degradation (Elbashir et al. 2001).

Degradation of mRNAs targeted by the RNAi pathway is initiated by endonucleolytic cleavage of the mRNA within the region complementary to the siRNA (Elbashir et al. 2001; Nykänen et al. 2001; Martinez et al. 2002). Structural and biochemical studies have provided compelling evidence that the endonucleolytic activity of RISC resides in the Argonaute proteins (AGO proteins), which constitute core components of RISC (Tabara et al. 1999; Hammond et al. 2001; Liu et al. 2004; Meister et al. 2004; Rand et al. 2004). Argonautes form a conserved family of highly basic proteins, characterized by a central PAZ domain and a C-terminal Piwi domain (Cerutti et al. 2000; Carmell et al. 2002). The PAZ domain binds to single-stranded $3^{\prime}$ ends of nucleic acids (Lingel et al. 2003, 2004; Song et al. 2003; Yan et al. 2003; Ma et al. 2004), and is involved in the specific recognition of the 2-nt 3' overhangs of siRNAs. The Piwi domain adopts an RNase H-like fold, and is thought to catalyze the endonucleolytic cleavage (Liu et al. 2004; Song et al. 2004).

Metazoan genomes encode more than one Argonaute protein (Carmell et al. 2002). There are four annotated paralogs in Drosophila, eight in human, and more than 20 in C. elegans. Despite their similar domain organization, not all Argonaute proteins are capable of mediating endonucleolytic cleavage. Indeed, in human and Drosophila cells, only AGO2-containing RISC is able to catalyze mRNA cleavage, even though all Argonaute proteins bind siRNAs (Liu et al. 2004; Meister et al. 2004; Okamura et al. 2004; Rand et al. 2004).

Following endonucleolytic cleavage by RISC, the mRNA is degraded by a mechanism that has not been elucidated. In Arabidopsis, mutations in the gene encoding one of the major cytoplasmic 5'-to-3' exonucleases (XRN4) leads to the stabilization of $3^{\prime}$ mRNA fragments derived from transcripts that are targets of endogenous miRNAs (Souret et al. 2004). That study suggested that mRNAs targeted by RISC are not degraded by a dedicated pathway, but are handed over to the general RNA decay machinery in the cell.

There are two major cytoplasmic pathways for degrading bulk mRNA in eukaryotes (for review, see Parker and Song 2004). Both pathways are initiated by shortening of the poly(A)-tail by deadenylases. In one pathway, deadenylation triggers decapping, and this exposes the mRNA body for $5^{\prime}$-to-3' digestion by the major cytoplasmic $5^{\prime}$-to- $3^{\prime}$ exonuclease XRN1. In yeast and mammalian cells, decay of mRNAs through this pathway is thought to occur in specialized cytoplasmic bodies or mRNA decay foci (also known as P-bodies or GW-bodies) where XRN1 and the decapping enzyme DCP2 colocalize (Ingelfinger et al. 2002; van Dijk et al. 2002; Eystathioy et al. 2003; Sheth and Parker 2003; Cougot et al. 2004).
In the second mRNA decay pathway, deadenylation is followed by $3^{\prime}$-to- $5^{\prime}$ degradation of the transcript. This requires the exosome, a multimeric assembly of $3^{\prime}$-to- $5^{\prime}$ exonucleases, and the Ski complex. The Ski complex was identified in yeast as a heterotrimeric protein complex (consisting of Ski2p, Ski3p, and Ski8p) that regulates exosome activity (Anderson and Parker 1998; Brown et al. 2000).

In addition to their role in general RNA decay, XRN1, the exosome, and the Ski complex function in mRNA surveillance pathways by which aberrant mRNAs are detected and degraded. These include the nonsense-mediated mRNA decay pathway (NMD), a pathway that eliminates mRNAs harboring premature translation termination codons (PTCs), and the nonstop decay (NSD) pathway that degrades mRNAs lacking a stop codon (for review, see Parker and Song 2004).

In this study we investigated the decay pathway of mRNAs targeted by RISC in Drosophila cells. In agreement with results reported by Okamura et al. (2004) and Rand et al. (2004), we show that endonucleolytic cleavage requires Drosophila AGO2, but not the additional Drosophila Argonaute paralogs (i.e., AGO1, PIWI, or Aubergine). Following the first AGO2-dependent cleavage, the resulting 5' fragment is degraded by the exosome, whereas the $3^{\prime}$ fragment is degraded by XRN1. Degradation of the $5^{\prime}$ fragment is prevented in cells depleted of Drosophila homologs of the yeast proteins Ski2p, Ski3p, and Ski8p, suggesting that the role of these proteins in $3^{\prime}$-to- $5^{\prime}$ mRNA decay is conserved.

\section{RESULTS}

\section{Generation of RNAi reporters for Drosophila cells}

To elucidate the mechanism by which mRNAs targeted by RISC are degraded, we made use of two previously described reporter constructs in which the coding region of the Drosophila alcohol dehydrogenase (adh) gene is placed downstream of the constitutive actin 5C promoter (constitutive adh reporter, 5C-adh, Fig. 1A), or downstream of a inducible metallothionein (Mtn) promoter (inducible adh reporter, Mtn-adh, Fig. 1A; Gatfield et al. 2003; Gatfield and Izaurralde 2004). We also designed an additional reporter in which the cDNA encoding human NXF1 is placed under the control of the Mtn promoter (Fig. 1A, Mtn-NXF1).

The reporter constructs were cotransfected with a plasmid encoding puromycin acetyl transferase into Drosophila Schneider cells (S2 cells) to generate polyclonal cell lines constitutively expressing adh, or expressing adh or human NXF1 after induction with copper sulfate. Cells were treated with dsRNAs targeting a central region of $\sim 300-450 \mathrm{nt}$ of adh or NXF1 mRNAs (Fig. 1A, black boxes). The steadystate levels of the transcripts were analyzed by Northern blot and normalized to those of the endogenous rp49 mRNA (encoding ribosomal protein L32). In cells treated with the specific dsRNAs, the normalized levels of the reporter tran- 
A

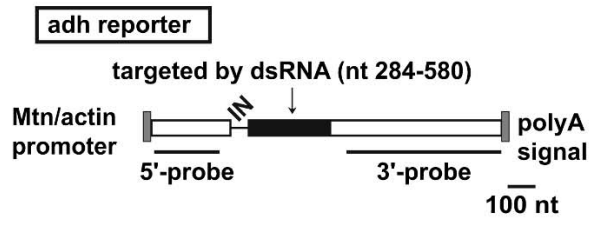

NXF1 reporter

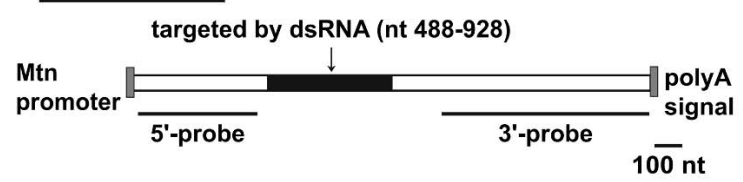

B 5C-adh

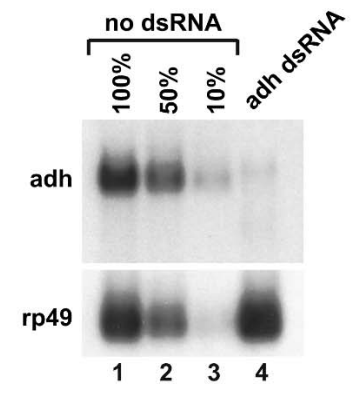

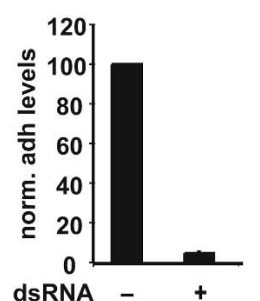

C Mtn-adh

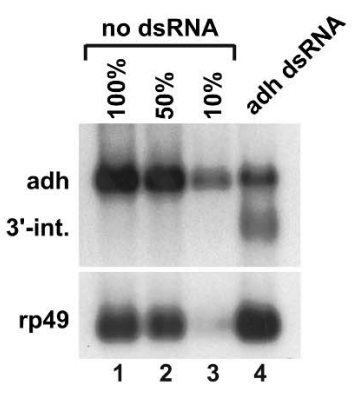

D Mtn-NXF1

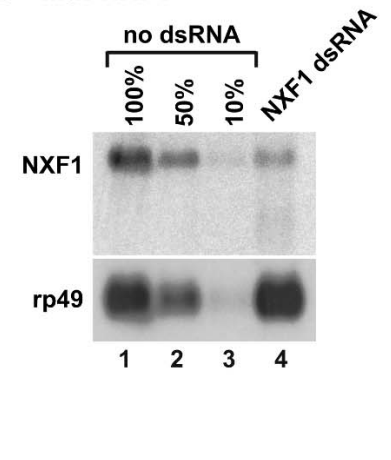

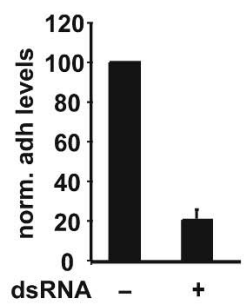

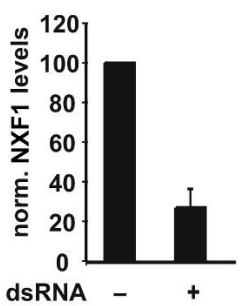

FIGURE 1. Generation of RNAi reporters for Drosophila cells. (A) Schematic representation of the reporters. White boxes, exons; gray boxes, sequences derived from vector pAc5.1b (Invitrogen) or pRmHa, respectively; black boxes, sequences complementary to the dsRNAs; IN, introns. The fragments of the transcript detected by the $5^{\prime}$ or $3^{\prime}$ probes used in this study are indicated. $(B-D)$ S2 cells expressing the indicated reporter constructs were treated with the corresponding dsRNAs. Total RNA samples were isolated and analyzed by Northern blot using probes specific for adh ( $3^{\prime}$ probe), NXF1 (3' probe), and rp49 mRNAs. In lanes 1-3, dilutions of total RNA samples isolated from untreated cells were loaded to assess the efficiency of RNAi. The levels of the reporters were quantitated in at least three independent experiments and normalized (norm.) to those of rp49 mRNA. These values were set to $100 \%$ in untreated cells. Mean values \pm standard deviations (SD) are shown.

scripts were reduced to $5 \%-25 \%$ of the levels detected in untreated cells (Fig. 1B-D), indicating that the reporter transcripts were effectively targeted by the dsRNAs.

We next investigated whether the reduction of mRNA levels caused by the dsRNAs was a consequence of increased mRNA turnover. In the experiment shown in Figure 2A-C, the half-life of the inducible adh reporter was determined after a short induction pulse followed by the inhibition of transcription by actinomycin D. In untreated cells, the halflife of Mtn-adh mRNA was longer than $4 \mathrm{~h}$, while in cells treated with adh dsRNA the half-life was $<15$ min (Fig. $2 \mathrm{~A}-\mathrm{C}$ ). Thus, the overall reduction in steady-state levels of the reporter transcripts targeted by dsRNAs is a direct consequence of a higher turnover rate.

To assess whether the reporters are degraded through the RNAi pathway, we investigated whether depletion of Argonaute proteins prevents dsRNA-mediated decay of the transcripts. S2 cells expressing 5C-adh reporter mRNA were treated with dsRNAs specific for AGO1, AGO2, PIWI, Aubergine (AUB), or GFP as a control. Four days after the first treatment, cells were treated again with the same dsRNAs and, in addition, adh dsRNA. The expression levels of adh mRNA were analyzed $3 \mathrm{~d}$ after the second transfection. In cells depleted of AGO2, adh dsRNA failed to reduce the expression levels of its cognate transcript (Fig. 2D, lane 4). In contrast, in cells depleted of AGO1, PIWI, or AUB, addition of adh dsRNA triggered a reduction of adh mRNA levels as efficiently as in control cells (Fig. 2D). Note that AGO1, AGO2, PIWI, and Aubergine are likely to be expressed in S2 cells, as the corresponding mRNAs are detectable by microarray analysis and RT-PCR (data not shown). Together, these observations suggest that only AGO2, but not AGO1, PIWI, or AUB, mediates siRNA-guided target cleavage in Drosophila, in agreement with Okamura et al. (2004) and Rand et al. (2004). These results also indicate that the mRNA reporters are degraded through the RNAi pathway in cells treated with the corresponding dsRNAs.

\section{3' mRNA fragments generated by RISC-mediated endonucleolytic cleavage are degraded by XRN1}

RISC initiates decay by cleaving the mRNA within a region complementary to the siRNA. This cleavage should generate a $5^{\prime}$ and a $3^{\prime}$ mRNA decay intermediate. In Arabidopsis, the $3^{\prime}$ decay intermediates are degraded by XRN4, a cytoplasmic 5'-to-3' exonuclease (Souret et al. 2004). The Drosophila genome encodes a single cytoplasmic $5^{\prime}$-to- $3^{\prime}$ exo- 
A Mtn-adh, no dsRNA

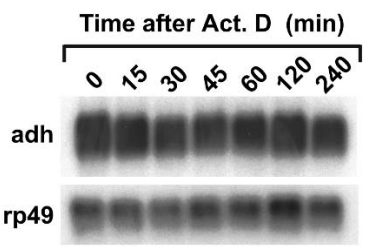

B Mtn-adh + adh dsRNA

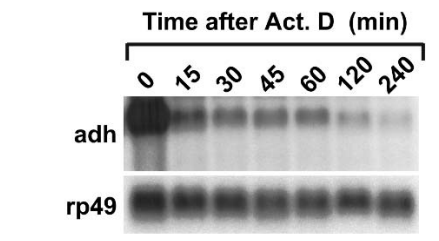

C

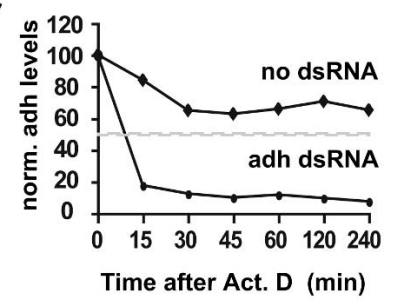

D 5 -adh
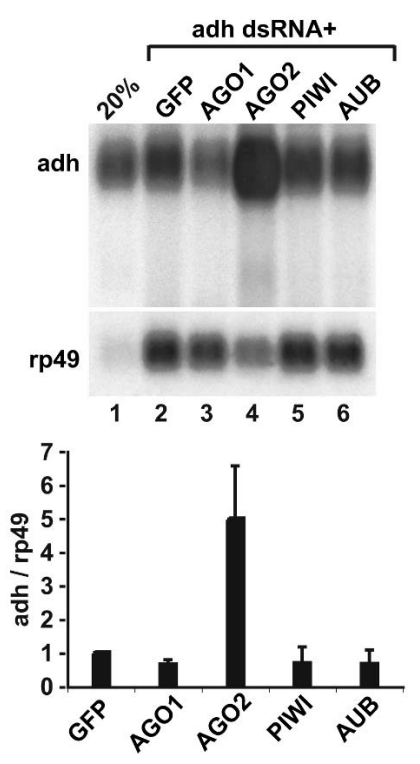

FIGURE 2. Reporter mRNAs are degraded through the RNAi pathway. $(A, B)$ S2 cells expressing Mtn-adh were treated with adh dsRNA. Expression of adh was induced for $45 \mathrm{~min}$ in treated and untreated cells. Following induction, transcription was inhibited by actinomycin D $(5 \mu \mathrm{g} / \mathrm{mL})$ for the times indicated above the lanes. Total RNA samples were isolated and analyzed as described in Figure 1. (C) The levels of adh mRNA normalized to those of rp49 mRNA shown in panels $A$ and $B$ are plotted as a function of time. (D) S2 cells constitutively expressing adh mRNA were treated with the dsRNAs indicated above the lanes. Total RNA samples were isolated and analyzed as described in Figure 1. The levels of the reporters were quantitated in at least three independent experiments and normalized (norm.) to those of rp49 mRNA. These values were set to 1 in cells treated with GFP and adh dsRNAs. Mean values \pm SD are shown.

nuclease known as XRN1 or Pacman. To investigate whether Drosophila XRN1 is responsible for the decay of the 3' mRNA fragment generated by RISC cleavage, we depleted the protein in cells constitutively expressing adh mRNA. When cells were also treated with adh dsRNA, depletion of XRN1 led to the accumulation of mRNA species with higher electrophoretic mobility (Fig. 3A, lane 3, 3 '-int.). These shorter RNA species were detected with a probe complementary to a region downstream of the dsRNA-target site, but not with a probe hybridizing upstream of the targeted region (see Figs. 3A, 5A below). The shorter RNA species were not observed when adh dsRNA was omitted (data not shown), and depletion of AGO2 prevented their appearance (Fig. 3A, lane 7), indicating that they represent $3^{\prime}$ decay intermediates that arose from the messages undergoing RNAi. Similar results were obtained for the Mtn-adh and Mtn-NXF1 reporters (Fig. $3 \mathrm{~B}, \mathrm{C})$.

To quantify the effect of the XRN1 depletion, the steadystate levels of the full-length transcripts and of the $3^{\prime}$ decay intermediates were quantitated in at least three independent experiments. The values obtained for the intermediate were divided by those obtained for the full-length transcript, in order to correct for potential nonspecific effects of the depletions and this ratio was set to a value of 1 in control cells (i.e., cells treated with GFP and adh dsRNAs). Although the $3^{\prime}$ fragments were also detected in control cells treated with the specific dsRNA (with or without addition of GFP dsRNA; Fig. 1B-D, lanes 4; Fig. 3A-C), their levels increase three- to eightfold in cells depleted of XRN1 (Fig. 3A-C). Depletion of the exosome components RRP4 or CSL4, or of SKI2 did not increase the levels of the $3^{\prime}$ fragments, indicating that these fragments are not degraded by deadenylation and $3^{\prime}$-to-5' exonucleolytic digestion by the exosome.

If the $3^{\prime}$ fragments were degraded by XRN1, one would expect the half-life of these fragments to be increased in cells depleted of XRN1 relative to cells treated with GFP dsRNA. Indeed, in cells treated with GFP and adh dsRNAs, the adh 3 ' intermediate had a half-life shorter than $15 \mathrm{~min}$, but was strongly stabilized in cells depleted of XRN1 (Fig. $4 \mathrm{~A}-\mathrm{C})$. We conclude that the $3^{\prime}$ mRNA fragments generated by RISC activity are degraded from their $5^{\prime}$ ends by XRN1.

Decay of the $5^{\prime}$ fragment is mediated by the exosome RISC-mediated endonucleolytic cleavage produces $5^{\prime}$ decay intermediates that have been observed both in vivo and in vitro (Elbashir et al. 2001; Nykänen et al. 2001; Martinez and Tuschl 2004; Schwarz et al. 2004), and could potentially be degraded by the exosome. In order to determine whether the exosome is indeed involved in this process, we silenced the expression of the exosome component RRP4 or CSL4 or the exosome cofactor SKI2 in cells expressing 5C-adh and treated with adh dsRNA. The effect of these depletions on the adh mRNA levels was analyzed by Northern blot and compared to the effects observed when GFP dsRNA was added. Depletion of RRP4, CSL4, or SKI2 led to the appearance of mRNA fragments that are detected using a probe spanning sequences $5^{\prime}$ to the region targeted by the dsRNA (Fig. 5, 5' int.). These fragments were not observed in the absence of adh dsRNA (data not shown) or in cells treated with AGO2 dsRNA (Fig. 5A, lane 7), indicating that they represent $5^{\prime}$ decay intermediates arising from the reporter targeted by RNAi. Similar results were seen with the Mtn-adh and NXF1 reporters (Fig. 5B,C). 
A 5 C-adh

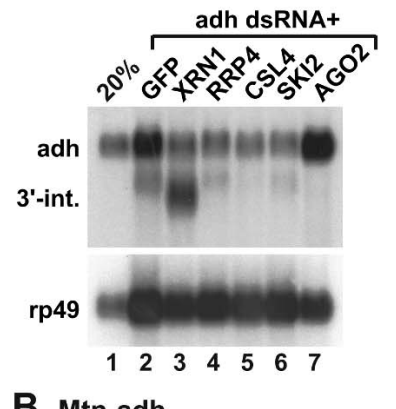

B Mtn-adh

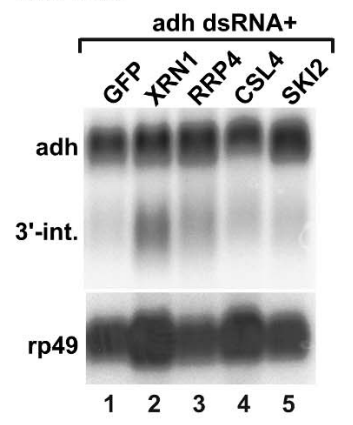

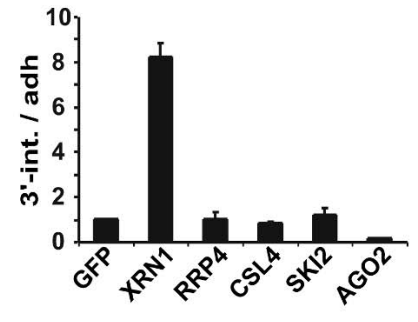

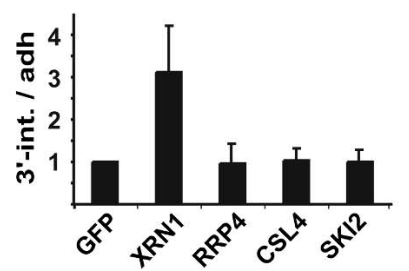

C Mtn-NXF1
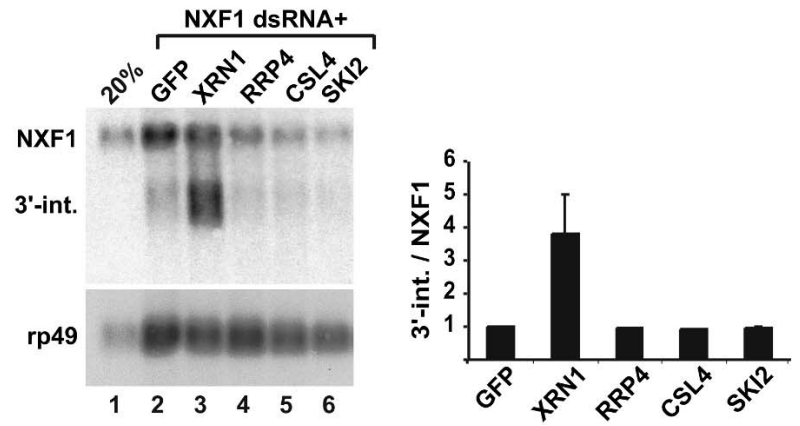

FIGURE 3. The $3^{\prime}$ fragments are degraded by XRN1. (A-C) S2 cells expressing 5C-adh, Mtn-adh, or Mtn-NXF1 were treated with the dsRNAs indicated above the lanes. Total RNA samples were analyzed as described in Figure 1. Northern blots were hybridized with probes complementary to the $3^{\prime}$ ends of the reporters as shown in Figure 1A. In lane 1 of panels $A$ and $C, 20 \%$ of an RNA sample isolated from untreated cells was loaded to assess the efficiency of RNAi. The levels of the 3 '-intermediate ( 3 '-int.) were normalized to those of the full-length mRNA in at least three independent experiments. These values were set to 1 in control cells treated with GFP and the specific (adh or NXF1) dsRNAs. Mean values \pm SD are shown.

Relative to the full-length transcript, the levels of the $5^{\prime}$ fragments increased three- to ninefold in cells depleted of RRP4, CSL4, or SKI2, but remained unchanged in cells depleted of XRN1 (Fig. 5A-C). Moreover, the half-life of the $5^{\prime}$ fragments was $<15 \mathrm{~min}$ in control cells, but increased to $60 \mathrm{~min}$ in cells depleted of RRP4 (Fig. 6A-C). We conclude that the $5^{\prime}$ mRNA fragments generated by RISC activity are degraded from their $3^{\prime}$ ends by the exosome.

\section{Drosophila homologs of Ski proteins are required for exosome-mediated decay of the $5^{\prime}$ intermediates}

In yeast, the Ski complex consists of Ski2p, Ski3p, and Ski8p (Brown et al. 2000). Although putative homologs of the Ski proteins are encoded by the higher eukaryotic genomes, it is unclear whether these proteins assemble in a complex with similar functions as in yeast. The observation that depletion of the Drosophila homolog of Ski2p [encoded by the gene twister (tst), also known as CG10210] leads to a strong stabilization of the $5^{\prime}$ decay intermediates (Fig. 5) prompted us to test whether the putative Drosophila homologs of Ski3p and Ski8p are also involved in this process. The closest homologs of Ski3p and Ski8p are encoded by the uncharacterized Drosophila genes CG8777 and CG3909, respectively.

In yeast, the interaction between the exosome and the Ski complex is thought to be mediated by the GTP-binding protein Ski7p (van Hoof et al. 2000; Araki et al. 2001). Ski7p is structurally related to eRF3 and Hbslp. Surprisingly, higher eukaryotic genomes encode orthologs of eRF3 and Hbs1, but no obvious orthologs of Ski7p. We therefore also tested whether Drosophila HBS1 could be the functional homolog of Ski7p.

In cells depleted of CG8777 or CG3909, but not of HBS1, the adh $5^{\prime}$ decay intermediate increased in abundance relative to the full-length transcript (Fig. 6D,E). These results argue that $t s t$ (or CG10210), CG8777, and CG3909 encode bona fide components of the Ski complex. Therefore we propose to call CG10210, CG8777, and CG3909 Drosophila ski2, ski3, and ski8, respectively.

\section{RISC-mediated decay of mRNA occurs independently of decapping and deadenylation}

The results described above suggest that the decay intermediates are degraded from the ends generated by RISC cleavage, without prior decapping or deadenylation. We therefore tested whether the $3^{\prime}$ fragments that accumulate in cells depleted of XRN1 are polyadenylated. If this were the case, we would expect these fragments to change their electrophoretic mobility upon removal of the poly(A)-tail by oligo(dT)-targeted ribonuclease $\mathrm{H}$ (RNase $\mathrm{H})$ cleavage. Both 


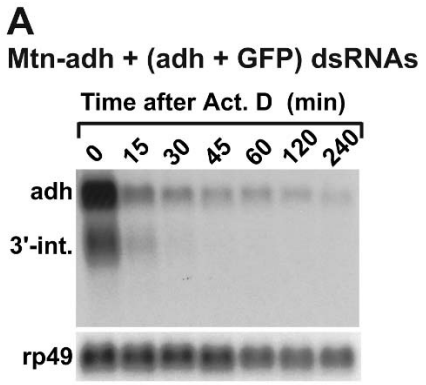

B

Mtn-adh + (adh + XRN1) dsRNAs

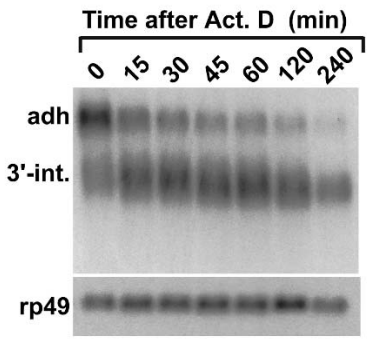

C

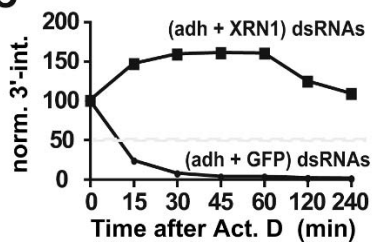

FIGURE 4. Decay rates of the $3^{\prime}$ fragments in cells depleted of XRN1. $(A, B)$ S2 cells expressing Mtn-adh were treated with the indicated dsRNAs. Expression of adh was induced for $45 \mathrm{~min}$. Transcription was inhibited by actinomycin $\mathrm{D}(5 \mu \mathrm{g} / \mathrm{mL})$ for the times indicated above the lanes. Total RNA samples were isolated and analyzed as described in Figure 1. Northern blots were hybridized with a $3^{\prime}$ probe (see Fig. 1A). (C) The levels of the $3^{\prime}$ fragments normalized to those of rp49 mRNA shown in panels $A$ and $B$ are plotted as a function of time.

the full-length transcript and the $3^{\prime}$ fragments change mobility following removal of the poly(A)-tail, indicating that these fragments are polyadenylated (Fig. 7A). In contrast, the $5^{\prime}$ fragments stabilized by depletion of SKI2 did not change mobility following RNase $\mathrm{H}$ treatment in the presence of oligo(dT) (Fig. 7B).

To test for the presence of a cap structure, we performed co-immunoprecipitations with antibodies recognizing the $5^{\prime}$ cap of mRNAs. We observed that full-length adh mRNA and the $5^{\prime}$ decay intermediates, but not the $3^{\prime}$ fragments, were preferentially co-immunoprecipitated with these antibodies (Fig. 7C,D). These results provide further evidence for a model in which the decay of mRNA fragments generated by RISC is independent of deadenylation and decapping.

\section{Efficient decay of the $5^{\prime}$ fragments requires ongoing translation}

The 5' fragments generated by RISC cleavage harbor a cap structure but lack a stop codon and a poly(A)-tail. As such, these mRNA fragments could be substrates for the nonstop decay (NSD) pathway. In yeast, this pathway requires the exosome, the Ski complex, and Ski7p (Frischmeyer et al. 2002; van Hoof et al. 2002). Moreover, mRNAs degraded through the NSD pathway are stabilized following inhibition of translation (Frischmeyer et al. 2002; van Hoof et al. 2002). To determine whether decay of the intermediates requires translation, cells constitutively expressing adh mRNA were treated with adh and GFP dsRNAs. As shown in Figures 3A and 5A (lane 2), this treatment leads to the appearance of the intermediates. Cells were then treated
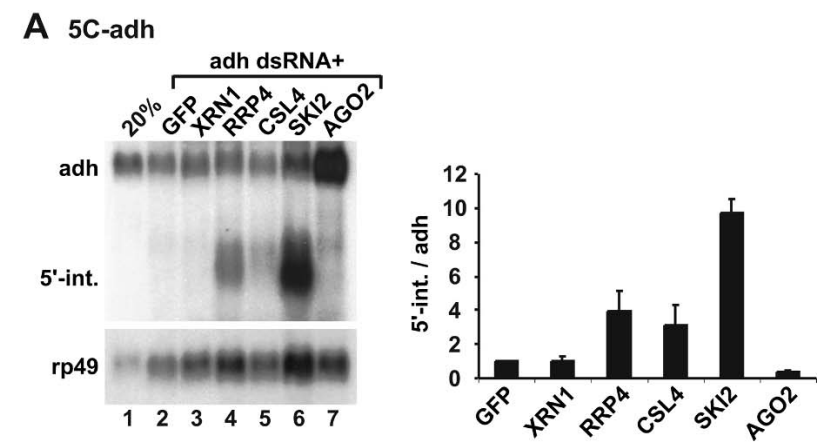

B Mtn-adh
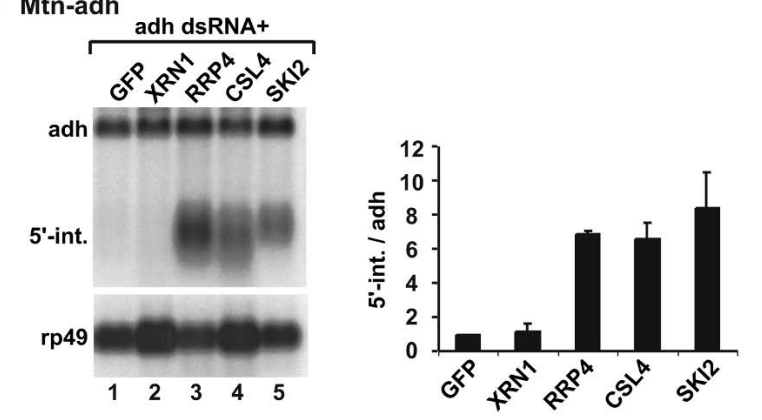

C Mtn-NXF1
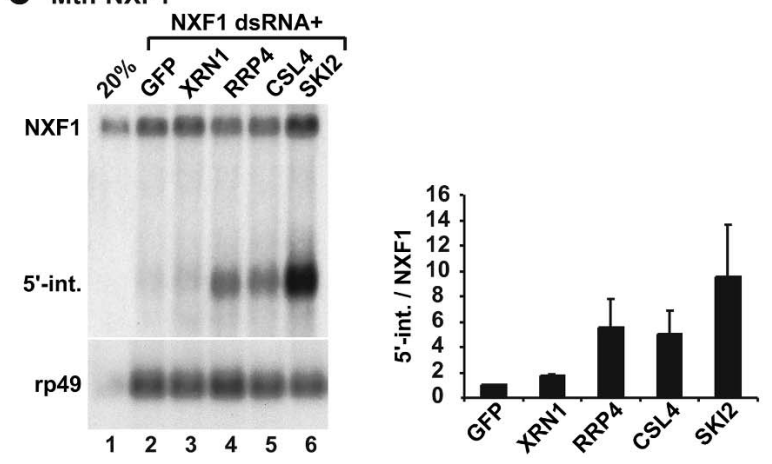

FIGURE 5. The $5^{\prime}$ fragments are degraded by the exosome. $(A-C)$ S2 cell-lines expressing $5 \mathrm{C}$-adh, Mtn-adh, or Mtn-NXF1 were treated with the dsRNAs indicated above the lanes. Total RNA samples were analyzed by Northern blot using probes detecting the $5^{\prime}$ decay intermediates (see Fig. 1A). The levels of the $5^{\prime}$ fragments were normalized to those of the full-length mRNA in at least three independent experiments. These values were set to 1 in control cells treated with GFP dsRNA and dsRNAs targeting the reporters. Mean values \pm SD are shown. In lane 1 of panels $A$ and $C$, a dilution of an RNA sample isolated from untreated cells was loaded to assess the efficiency of RNAi. 
A

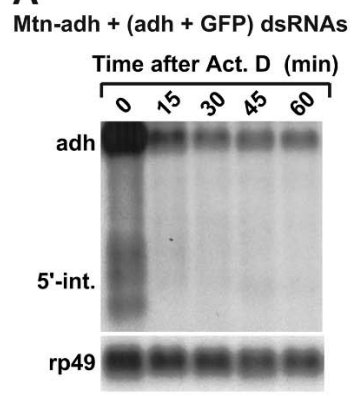

C

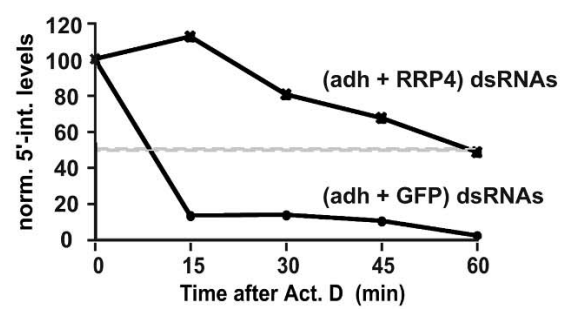

B

Mtn-adh + (adh + RRP4) dsRNAs

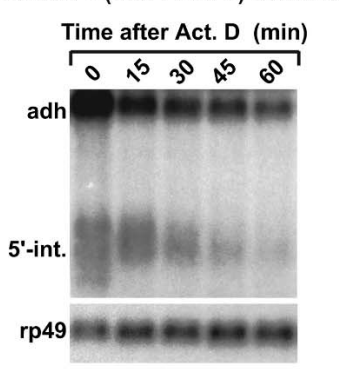

D 5C-adh

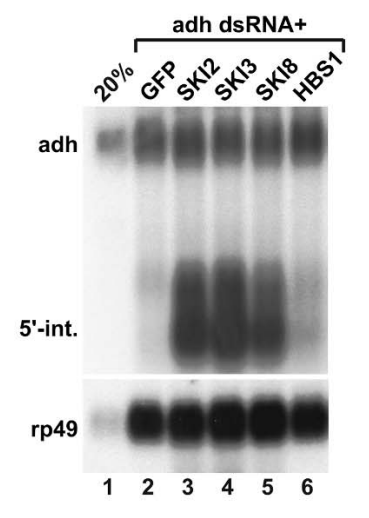

E

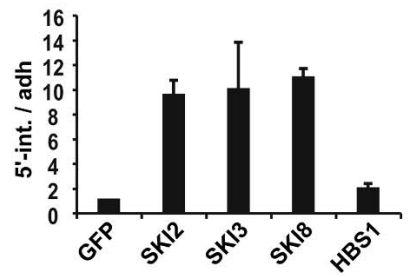

FIGURE 6. Degradation of the $5^{\prime}$ decay intermediates. $(A, B)$ S2 cell-lines expressing Mtn-adh were treated with adh and RRP4 dsRNAs. Expression of adh mRNA was induced for $45 \mathrm{~min}$. Next, transcription was inhibited with actinomycin D $(5 \mu \mathrm{g} / \mathrm{mL})$ for the times indicated above the lanes. Total RNA samples were isolated and analyzed by Northern blot with a $5^{\prime}$ probe. $(C)$ The levels of the $5^{\prime}$ fragments normalized to those of rp49 mRNA shown in panels $A, B$ are plotted as a function of time. (D) S2 cell lines expressing 5C-adh were transfected with the dsRNAs indicated above the lanes. RNA samples were analyzed by Northern blot, as described in Figure 1 . The steady-state levels of the $5^{\prime}$ decay intermediates were quantitated and normalized to those of the full-length transcript.

with cycloheximide for $45 \mathrm{~min}$, and the steady-state levels of the intermediates were quantitated and normalized to those of the full-length mRNA. In the presence of cycloheximide, the levels of the $5^{\prime}$ fragment increased three- to fivefold relative to the levels of rp49 (or adh) mRNAs, whereas the levels of the $3^{\prime}$ fragments remained unchanged (Fig. 8A-C). This suggests that efficient decay of the $5^{\prime}$ fragment requires ongoing translation.

\section{DISCUSSION}

In this study we analyzed the pathway by which mRNAs targeted by RNAi are degraded. We show that following siRNA-guided endonucleolytic cleavage by RISC, the resulting $5^{\prime}$ and $3^{\prime}$ mRNA fragments are degraded from the ends generated by RISC activity without decapping or deadenylation. Mechanistic studies of RISC-mediated cleavage have revealed that the reaction requires $\mathrm{Mg}^{2+}$ ions and leaves $3^{\prime}-\mathrm{OH}$ and $5^{\prime}$ phosphate termini (Martinez and Tuschl 2004; Schwarz et al. 2004). This is compatible with the observation that the $5^{\prime}$ and $3^{\prime}$ RNA fragments generated by RISC-cleavage are degraded by the exosome and XRN1, respectively. Indeed, the exosome has a strong preference for RNAs with 3' hydroxyl groups (Mitchell et al. 1997), and RNAs having $5^{\prime}$ phosphate groups are the preferred substrates of XRN1 (Stevens 2001).

The decay pathway of mRNAs targeted by RISC parallels nonsense-mediated mRNA decay (NMD) in Drosophila. Indeed, in this organism, degradation of PTC-containing messages is initiated by endonucleolytic cleavage in the vicinity of the nonsense codon (Gatfield and Izaurralde 2004). The resulting $5^{\prime}$ fragments are rapidly degraded by the exosome, and this requires at least SKI2, while the $3^{\prime}$ fragments are degraded by XRN1 (Gatfield and Izaurralde 2004). Notably, the $5^{\prime}$ fragments derived from mRNAs undergoing NMD or RNAi may also enter the nonstop decay (NSD) pathway, as ribosomes engaged in translating these fragments would be stalled at their $3^{\prime}$ ends. This raises the question of how the mRNA surveillance and RNAi machineries interact with general RNA decay enzymes and recruit them specifically to their targeted mRNAs.

Finally, the accumulation of the mRNA fragments flanking the region targeted by dsRNAs in cells depleted of the exosome or XRN1 confirms the observation that in Drosophila there is no amplification of the silencing triggers, and only the region complementary to the dsRNA is cleaved by RISC (Schwarz et al. 2002; Roignant et al. 2003).

\section{Drosophila SKI2, SKI3, and SKI8 are required for exosome-mediated decay of the $5^{\prime}$ intermediates}

The yeast proteins Ski2p, Ski3p, and Ski8p form a complex required for exosome-mediated $3^{\prime}$-to-5' mRNA decay in yeast (Anderson and Parker 1998; Brown et al. 2000). The precise mechanism by which these proteins regulate exosome activity is unknown. Ski2p is a putative RNA helicase of the DEVH family (Widner and Wickner 1993). Ski3p is characterized by the presence of 10 tetratricopeptide repeats 
A

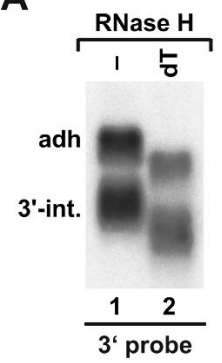

C

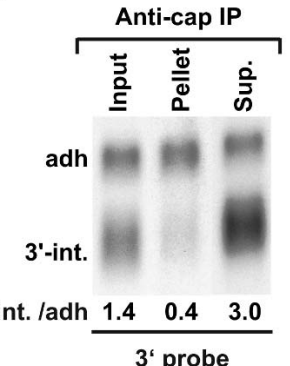

B

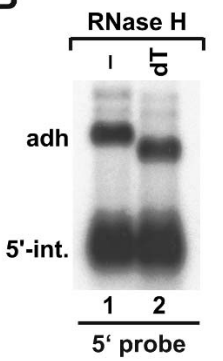

D

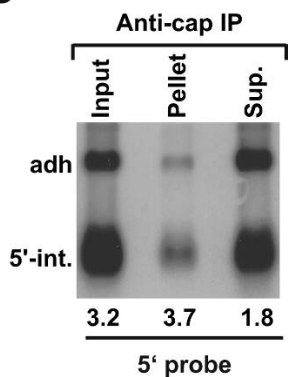

FIGURE 7. The $5^{\prime}$ intermediates are capped, whereas the $3^{\prime}$ intermediates are polyadenylated. Cells expressing the inducible adh reporter were codepleted of SKI2 and XRN1. In $(A, B)$, total RNA samples were subjected to oligo(dT)-targeted RNase $\mathrm{H}$ cleavage. In $(C, D)$, total RNA samples were co-immunoprecipitated with anti-cap antibodies. Northern blots were hybridized with $5^{\prime}$ or $3^{\prime}$ probes as indicated. The positions of the $3^{\prime}$ and $5^{\prime}$ intermediates are shown. In $C$, one-tenth of the inputs and supernatants and $100 \%$ of the immunoprecipitates were analyzed by Northern blot. In $D, 100 \%$ of the inputs, supernatants, and pellets was loaded. The levels of the intermediates relative to those of the full-length transcript are indicated below the lanes.

(TPRs; Rhee et al. 1989), 34-amino-acid $\alpha$-helical repeats known to mediate protein:protein interactions (D'Andrea and Regan 2003). Ski8p (also known as Rec14) folds into a seven-bladed propeller, a fold thought to serve as a scaffold for the assembly of multimeric protein complexes (Cheng et al. 2004; Madrona and Wilson 2004). In addition to its role in mRNA decay, Ski8p plays a role in the formation of meiotic DNA double-strand breaks and associates with chromosomes during meiosis (Arora et al. 2004). These two roles of Ski8p are conserved in fungi, but can be genetically separated (Evans et al. 1997; Tessé et al. 2003; Arora et al. 2004).

Putative homologs of the yeast Ski proteins have been identified in metazoa, but whether they have similar functions as in yeast remains unresolved. In this regard, human Ski2 (known as Ski2w) is mainly nucleolar (Qu et al. 1998) while yeast Ski2p is predominantly cytoplasmic (Brown et al. 2000), suggesting divergent functions. In this study we characterized the components of the putative Ski complex in Drosophila. We show that SKI2, SKI3, and SKI8 are all required for exosome-mediated decay of $5^{\prime}$ mRNA fragments generated by RISC cleavage.

The observation that degradation of the $5^{\prime}$ intermediates is prevented in cells depleted of SKI2, SKI3, or SKI8, despite the presence of the exosome, suggests that these proteins are essential for exosome activity or for its recruitment to the mRNA, as has been suggested for their yeast homologs (Anderson and Parker 1998; van Hoof et al. 2000; Araki et al. 2001). Further biochemical and functional studies are required to determine the precise composition and role of the Ski complex in metazoa.

In addition to the exosome and the Ski complex, the yeast protein Ski7p is also required for $3^{\prime}$-to-5' mRNA decay and has been implicated in both the NMD and the NSD pathways (van Hoof et al. 2000, 2002; Araki et al. 2001; Taka-

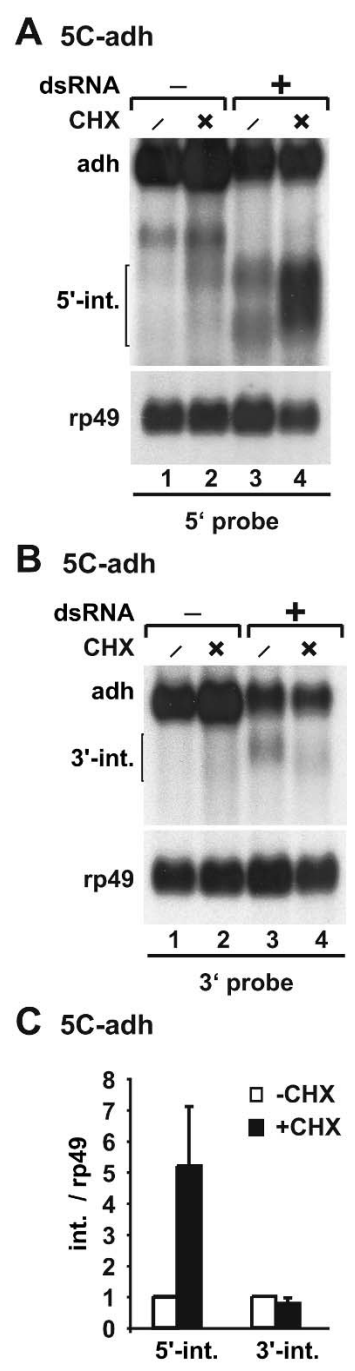

FIGURE 8. Degradation of the $5^{\prime}$ fragments requires ongoing translation. $(A, B)$ Cells expressing $5 \mathrm{C}$-adh were transfected with adh (and GFP) dsRNA as indicated (lanes 3,4). Translation was inhibited with cycloheximide (CHX, $100 \mu \mathrm{g} / \mathrm{mL}$ ) for $45 \mathrm{~min}$ (lanes 2,4). Total RNA samples were isolated and analyzed by Northern blot with probes detecting the $5^{\prime}$ or the $3^{\prime}$ decay intermediates. Lanes 1 and 2 show that the intermediates are not observed in treated $(\mathrm{CHX}+)$ or untreated $(\mathrm{CHX}-)$ cells in the absence of adh dsRNA. (C) The levels of the intermediates were normalized to those of the rp49 mRNA in at least three independent experiments. These values were set to 1 in untreated cells $(-\mathrm{CHX})$. Mean values \pm SD are shown. 
hashi et al. 2003). Ski7p interacts with the exosome and the Ski complex via its unique $\mathrm{N}$-terminal domain (Araki et al. 2001). This domain is sufficient for general $3^{\prime}$-to- $5^{\prime}$ mRNA degradation (Araki et al. 2001). In contrast, the C-terminal domain of Ski7p, which is structurally related to the GTPase domains of EF1A, eRF3 and Hbs1p, is dispensable for $3^{\prime}$ to-5' mRNA decay but is necessary for decay of transcripts lacking a stop codon (Araki et al. 2001; van Hoof et al. 2002). These observations lead to a model in which the C-terminal domain of Ski7p binds to the A site of the ribosomes stalled at the $3^{\prime}$-end of nonstop mRNAs, while the $\mathrm{N}$-terminal domain would recruit the exosome and the Ski complex to target the aberrant transcript for decay.

Surprisingly, there are no obvious orthologs of Ski7p in higher eukaryotes. This has raised the possibility that HBS1, the closest homolog of Ski7p, could have evolved additional functions in metazoa. However, we show that Drosophila HBS1 is not involved in decay of mRNA fragments generated by RISC. Similarly, Drosophila HBS1 is not implicated in the decay of mRNAs degraded by the NMD pathway (D. Gatfield and E. Izaurralde, unpubl.). It would be of interest to determine how ribosomes translating mRNA fragments lacking termination codons are released from the $3^{\prime}$-end of the transcript in higher eukaryotes.

\section{Recruitment of decay enzymes and recycling of RISC}

RISC can catalyze multiple rounds of mRNA cleavage (Hutvágner and Zamore 2002). How RISC dissociates from the mRNA after cleavage is unknown. Presumably, this process involves unwinding of the siRNA/mRNA duplexes. Unwinding would also be required to release the mRNA fragments, so that their ends are exposed to the exonucleolytic activities of the exosome and XRN1. Several RNA helicases have been implicated in RNAi, but with the exception of Armitage, their precise role in this process remains unresolved (for review, see Meister and Tuschl 2004). Alternatively, RISC release could be facilitated or may require degradation of the mRNA products. In this regard, RISC components may interact with factors involved in mRNA decay and promote their recruitment to the targeted mRNA. It is also possible that RISC components remain bound to the $3^{\prime}$ decay intermediates and escort them to P-bodies where the intermediates decay rapidly and the RISC complex would dissociate and be recycled. Whether decay of the $3^{\prime}$ mRNA fragments generated by RISC occurs in the cytoplasm or in mRNA decay bodies, however, remains to be established.

\section{MATERIALS AND METHODS}

\section{Construction of RNAi reporters}

The adh reporters have been described (Gatfield et al. 2003; Gatfield and Izaurralde 2004). To generate the NXF1 reporters, a cDNA fragment encoding human NXF1 was cloned into the
EcoRI-SalI sites of vector pRmHa containing the metallothionein promoter (Bunch et al. 1988).

\section{Cell culture and RNA interference}

Establishment of stable cell lines expressing the RNAi reporters and RNAi were performed as described (Gatfield et al. 2003; Gatfield and Izaurralde 2004); dsRNAs corresponding to Drosophila XRN1 (pcm, CG3291), RRP4 (CG3931), CSL4 (CG6249), SKI2 (tst, CG10210), SKI3 (CG8777), SKI8 (CG3909), and HBS1 (CG1898) were transcribed in vitro from $\sim 700$-bp cDNA fragments amplified from a Schneider cell cDNA library.

\section{RNA analysis}

RNA preparation and Northern blot analysis were performed essentially as described by Gatfield et al. (2003). RNAs from inducible cell lines were harvested $45 \mathrm{~min}$ after induction with $0.5 \mathrm{mM}$ copper sulfate. For determination of RNA half-lives, control or depleted cells were incubated with actinomycin $\mathrm{D}(5 \mu \mathrm{g} / \mathrm{mL})$ for the times indicated in the figures. Total RNA samples were analyzed by Northern blot. The levels of the reporters and intermediates were quantitated and normalized to the levels of rp49 mRNA (whose levels relative to $18 \mathrm{~S}$ rRNA do not change within the time-frame of the experiments).

Body-labeled DNA probes were generated by unidirectional PCR or random priming according to standard protocols. RNase $\mathrm{H}$ (USB) digestion using a $(\mathrm{dT})_{15}$ oligonucleotide was performed according to the manufacturer's instructions.

Immunoprecipitation of capped mRNAs was performed from total RNA preparations using the monoclonal antibody $\mathrm{H} 20$ in NET buffer (50 mM Tris-HCl pH 7.5, $150 \mathrm{mM} \mathrm{NaCl,} \mathrm{0.1 \%} \mathrm{NP40,}$ $1 \mathrm{mM}$ EDTA).

\section{ACKNOWLEDGMENTS}

We thank David Gatfield and Kevin Czaplinski for helpful discussions and Reinhard Lührmann for the anti-cap antibodies. This study was supported by the European Molecular Biology Organisation (EMBO) and the Human Frontier Science Program Organization (HFSPO). T.I.O. is a recipient of the Hungarian State Eötvös fellowship.

Received November 9, 2004; accepted December 20, 2004.

\section{REFERENCES}

Ambros, V. 2004. The functions of animal microRNAs. Nature 431: 350-355.

Anderson, J.S. and Parker, R.P. 1998. The $3^{\prime}$ to $5^{\prime}$ degradation of yeast mRNAs is a general mechanism for mRNA turnover that requires the SKI2 DEVH box protein and $3^{\prime}$ to $5^{\prime}$ exonucleases of the exosome complex. EMBO J. 17: 1497-1506.

Araki, Y., Takahashi, S., Kobayashi, T., Kajiho, H., Hoshino, S., and Katada, T. 2001. Ski7p G protein interacts with the exosome and the Ski complex for $3^{\prime}$-to-5' mRNA decay in yeast. EMBO J. 20: 4684-4693.

Arora, C., Kee, K., Maleki, S., and Keeney, S. 2004. Antiviral protein Ski8 is a direct partner of Spo11 in meiotic DNA break formation, independent of its cytoplasmic role in RNA metabolism. Mol. Cell 
13: 549-559.

Baulcombe D. 2004. RNA silencing in plants. Nature 409: 356-363.

Bernstein, E., Caudy, A.A., Hammond, S.M., and Hannon, G.J. 2001. Role for a bidentate ribonuclease in the initiation step of RNA interference. Nature 409: 363-366.

Brown, J.T., Bai, X., and Johnson, A.W. 2000. The yeast antiviral proteins Ski2p, Ski3p, and Ski8p exist as a complex in vivo. RNA 6: 449-457.

Bunch, T. A., Grinblat, Y., and Goldstein, L.S. 1988. Characterization and use of the Drosophila metallothionein promoter in cultured Drosophila melanogaster cells. Nucleic Acids Res. 16: 1043-1061.

Carmell, M.A., Xuan, Z., Zhang, M.Q., and Hannon, G.J. 2002. The Argonaute family: Tentacles that reach into RNAi, developmental control, stem cell maintenance, and tumorigenesis. Genes \& Dev. 16: $2733-2742$.

Cerutti, L., Mian, N., and Bateman, A. 2000. Domains in gene silencing and cell differentiation proteins: The novel PAZ domain and redefinition of the Piwi domain. Trends Biochem. Sci. 25: 481-482.

Cheng, Z., Liu, Y., Wang, C., Parker, R., and Song, H. 2004. Crystal structure of Ski8p, a WD-repeat protein with dual roles in mRNA metabolism and meiotic recombination. Protein Sci. 13: 26732684.

Cougot, N., Babajko, S., and Seraphin, B. 2004. Cytoplasmic foci are sites of mRNA decay in human cells. J. Cell Biol. 165: 31-40.

D'Andrea, L.D. and Regan, L. 2003. TPR proteins: The versatile helix. Trends Biochem. Sci. 28: 655-662.

Elbashir, S.M., Lendeckel, W., and Tuschl, T. 2001. RNA interference is mediated by 21- and 22-nucleotide RNAs. Genes \& Dev. 15: 188200.

Evans, D.H., Li, Y.F., Fox, M.E., and Smith, G.R. 1997. A WD repeat protein, Rec14, essential for meiotic recombination in Schizosaccharomyces pombe. Genetics 146: 1253-1264.

Eystathioy, T., Jakymiw, A., Chan, E.K., Seraphin, B., Cougot, N., and Fritzler, M.J. 2003. The GW182 protein colocalizes with mRNA degradation associated proteins hDcp1 and hLSm4 in cytoplasmic GW bodies. RNA 9: 1171-1173.

Frischmeyer, P.A., van Hoof, A., O’Donnell, K., Guerrerio, A.L., Parker, R., and Dietz, H.C. 2002. An mRNA surveillance mechanism that eliminates transcripts lacking termination codons. Science 295: 2258-2261.

Gatfield, D. and Izaurralde, E. 2004. Nonsense-mediated messenger RNA decay is initiated by endonucleolytic cleavage in Drosophila. Nature 429: 575-578.

Gatfield, D., Unterholzner, L., Ciccarelli, F.D., Bork, P., and Izaurralde, E. 2003. Nonsense-mediated mRNA decay in Drosophila: At the intersection of the yeast and mammalian pathways. EMBO J. 22: 3960-3970.

Grishok, A., Pasquinelli, A.E., Conte, D., Li, N., Parrish, S., Ha, I., Baillie, D.L., Fire, A., Ruvkun, G., and Mello, C.C. 2001. Genes and mechanisms related to RNA interference regulate expression of the small temporal RNAs that control C. elegans developmental timing. Cell 106: 23-34.

Hammond, S.M., Boettcher, S., Caudy, A.A., Kobayashi, R., and Hannon, G.J. 2001. Argonaute2, a link between genetic and biochemical analyses of RNAi. Science 293: 1146-1150.

Hutvágner, G. and Zamore, P.D. 2002. A microRNA in a multipleturnover RNAi enzyme complex. Science 297: 2056-2060.

Hutvágner, G., McLachlan, J., Pasquinelli, A.E., Balint, E., Tuschl, T., and Zamore, P.D. 2001. A cellular function for the RNA-interference enzyme Dicer in the maturation of the let-7 small temporal RNA. Science 293: 834-838.

Ingelfinger, D., Arndt-Jovin, D.J., Luhrmann, R., and Achsel, T. 2002. The human LSm1-7 proteins colocalize with the mRNA-degrading enzymes Dcp1/2 and Xrnl in distinct cytoplasmic foci. RNA 8: $1489-1501$.

Lingel, A., Simon, B., Izaurralde, E., and Sattler, M. 2003. Structure and nucleic-acid binding of the Drosophila Argonaute 2 PAZ domain. Nature 426: 465-469.

- 2004. Nucleic acid 3'-end recognition by the Argonaute2 PAZ domain. Nat. Struct. \& Mol. Biol. 11: 576-577.

Lippman, Z. and Martienssen, R. 2004. The role of RNA interference in heterochromatic silencing. Nature 431: 364-370.

Liu, J., Carmell, M.A., Rivas, F.V., Marsden, C.G., Thomson, J.M., Song, J.J., Hammond, S.M., Joshua-Tor, L., and Hannon, G.J. 2004. Argonaute2 is the catalytic engine of mammalian RNAi. Science 305: 1437-1441.

Ma, J.B., Ye, K., and Patel, D.J. 2004. Structural basis for overhangspecific small interfering RNA recognition by the PAZ domain. Nature 429: 318-322.

Madrona, A.Y. and Wilson, D.K. 2004. The structure of Ski8p, a protein regulating mRNA degradation: Implications for WD protein structure. Protein Sci. 13: 1557-1565.

Martinez, J. and Tuschl, T. 2004. RISC is a $5^{\prime}$ phosphomonoesterproducing RNA endonuclease. Genes \& Dev. 18: 975-980.

Martinez, J., Patkaniowska, A., Urlaub, H., Luhrmann, R., and Tuschl, T. 2002. Single-stranded antisense siRNAs guide target RNA cleavage in RNAi. Cell 110: 563-574.

Meister, G. and Tuschl, T. 2004. Mechanisms of gene silencing by double-stranded RNA. Nature 431: 343-349.

Meister, G., Landthaler, M., Patkaniowska, A., Dorsett, Y., Teng, G., and Tuschl, T. 2004. Human Argonaute2 mediates RNA cleavage targeted by miRNAs and siRNAs. Mol. Cell 15: 185-197.

Mitchell, P., Petfalski, E., Shevchenko, A., Mann, M., and Tollervey, D. 1997. The exosome: A conserved eukaryotic RNA processing complex containing multiple $3^{\prime} \rightarrow 5^{\prime}$ exoribonucleases. Cell 91: 457466.

Nykänen, A., Haley, B., and Zamore, P.D. 2001. ATP requirements and small interfering RNA structure in the RNA interference pathway. Cell 107: 309-321.

Okamura, K., Ishizuka, A., Siomi, H., and Siomi, M.C. 2004. Distinct roles for Argonaute proteins in small RNA-directed RNA cleavage pathways. Genes \& Dev. 18: 1655-1666.

Parker, R. and Song, H. 2004. The enzymes and control of eukaryotic mRNA turnover. Nat. Struct.\& Mol. Biol. 11: 121-127.

Plasterk, R.H. 2002. RNA silencing: The genome's immune system. Science 296: 1263-1265.

Qu, X., Yang, Z., Zhang, S., Shen, L., Dangel, A.W., Hughes, J.H., Redman, K.L., Wu, L.C., and Yu, C.Y. 1998. The human DEVHbox protein Ski2w from the HLA is localized in nucleoli and ribosomes. Nucleic Acids Res. 26: 4068-4077.

Rand, T.A., Ginalski, K., Grishin, N.V., and Wang, X. 2004. Biochemical identification of Argonaute 2 as the sole protein required for RNA-induced silencing complex activity. Proc. Natl. Acad. Sci. 101: 14385-14389.

Rhee, S.K., Icho, T., and Wickner, R.B. 1989. Structure and nuclear localization signal of the SKI3 antiviral protein of Saccharomyces cerevisiae. Yeast 5: 149-158.

Roignant, J.Y., Carre, C., Mugat, B., Szymczak, D., Lepesant, J.A., and Antoniewski, C. 2003. Absence of transitive and systemic pathways allows cell-specific and isoform-specific RNAi in Drosophila. RNA 9: 299-308.

Schwarz, D.S., Hutvagner, G., Haley, B., and Zamore, P.D. 2002. Evidence that siRNAs function as guides, not primers, in the Drosophila and human RNAi pathways. Mol. Cell 10: 537-548.

Schwarz, D.S., Tomari, Y., and Zamore, P.D. 2004. The RNA-induced silencing complex is a $\mathrm{Mg} 2+-$ dependent endonuclease. Curr. Biol. 14: 787-791.

Sheth, U. and Parker, R. 2003. Decapping and decay of messenger RNA occur in cytoplasmic processing bodies. Science 300: 805-808.

Song, J.J., Liu, J., Tolia, N.H., Schneiderman, J., Smith, S.K., Martienssen, R.A., Hannon, G.J., and Joshua-Tor, L. 2003. The crystal structure of the Argonaute2 PAZ domain reveals an RNA binding motif in RNAi effector complexes. Nat. Struct. Biol. 10: 1026-1032.

Song, J.J., Smith, S.K., Hannon, G.J., and Joshua-Tor, L. 2004. Crystal structure of Argonaute and its implications for RISC slicer activity. Science 305: 1434-1437.

Souret, F.F., Kastenmayer, J.P., and Green, P.J. 2004. AtXRN4 degrades mRNA in Arabidopsis and its substrates include selected 
miRNA targets. Mol. Cell 15: 173-183.

Stevens, A. 2001. 5'-exoribonuclease 1: Xrn1. Methods Enzymol. 342: 251-259.

Tabara, H., Sarkissian, M., Kelly, W.G., Fleenor, J., Grishok, A., Timmons, L., Fire, A., and Mello, C.C. 1999. The rde-1 gene, RNA interference, and transposon silencing in C. elegans. Cell 99: 123132.

Takahashi, S., Araki, Y., Sakuno, T., and Katada, T. 2003. Interaction between Ski7p and Upflp is required for nonsense-mediated $3^{\prime}$ to-5' mRNA decay in yeast. EMBO J. 22: 3951-3959.

Tessé, S., Storlazzi, A., Kleckner, N., Gargano, S., and Zickler, D. 2003. Localization and roles of Ski8p protein in Sordaria meiosis and delineation of three mechanistically distinct steps of meiotic homolog juxtaposition. Proc. Natl. Acad. Sci. 100: 12865-12870.

van Dijk, E., Cougot, N., Meyer, S., Babajko, S., Wahle, E., and Seraphin, B. 2002. Human Dcp2: A catalytically active mRNA de- capping enzyme located in specific cytoplasmic structures. $E M B O$ J. 21: 6915-6924.

van Hoof, A., Staples, R.R., Baker, R.E., and Parker, R. 2000. Function of the ski4p (Csl4p) and Ski7p proteins in $3^{\prime}-$ to-5' degradation of mRNA. Mol. Cell. Biol. 20: 8230-8243.

van Hoof, A., Frischmeyer, P.A., Dietz, H.C., and Parker, R. 2002. Exosome-mediated recognition and degradation of mRNAs lacking a termination codon. Science 295: 2262-2264.

Widner, W.R. and Wickner, R.B. 1993. Evidence that the SKI antiviral system of Saccharomyces cerevisiae acts by blocking expression of viral mRNA. Mol. Cell. Biol. 13: 4331-4341.

Williams, B.R. 1999. PKR; a sentinel kinase for cellular stress. Oncogene 18: 6112-6120.

Yan, K.S., Yan, S., Farooq, A., Han, A., Zeng, L., and Zhou, M.M. 2003. Structure and conserved RNA binding of the PAZ domain. Nature 426: 468-474. 

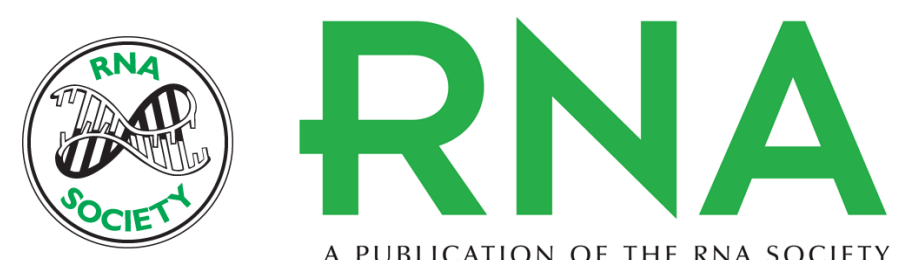

A PUBLICATION OF THE RNA SOCIETY

\section{Decay of mRNAs targeted by RISC requires XRN1, the Ski complex, and the exosome}

TAMAS I. ORBAN and ELISA IZAURRALDE

RNA 2005 11: 459-469

References This article cites 59 articles, 27 of which can be accessed free at: http://rnajournal.cshlp.org/content/11/4/459.full.html\#ref-list-1

\section{License}

Email Alerting Service

Receive free email alerts when new articles cite this article - sign up in the box at the top right corner of the article or click here. 